\title{
Aluminum ion parameters for the 2015 PP-on-Au setup in RHIC
}

\author{
C. J. Gardner
}

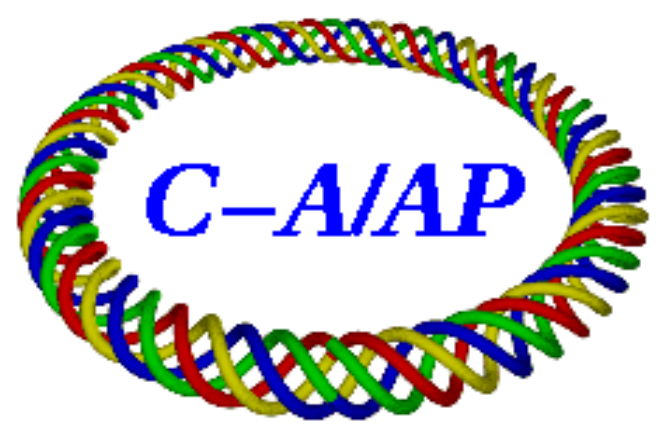

\section{Collider-Accelerator Department Brookhaven National Laboratory Upton, NY 11973}

\section{U.S. Department of Energy \\ Office of Science, Office of Nuclear Physics}

Notice: This document has been authorized by employees of Brookhaven Science Associates, LLC under Contract No. DE-SC0012704 with the U.S. Department of Energy. The United States Government retains a nonexclusive, paid-up, irrevocable, world-wide license to publish or reproduce the published form of this document, or allow others to do so, for United States Government purposes. 


\title{
Aluminum Ion Parameters for the 2015 PP-on-Al Setup in RHIC
}

\author{
C.J. Gardner
}

October 2, 2015

In this note the nominal parameters for aluminum ions in Booster, AGS, and RHIC are given for the PP-on-Al setup in RHIC.

The setup parameters are summarized in Sections 13, 14, 15.

\section{Mass}

An aluminum ion with charge $e Q$ has $N=14$ neutrons, $Z=13$ protons, and $(Z-Q)$ electrons. Here $Q$ is an integer and $e$ is the charge of a single proton. The mass number is

$$
A=N+Z=27 .
$$

This is also called the number of nucleons. The mass energy equivalent of the ion is

$$
m c^{2}=a m_{u} c^{2}-Q m_{e} c^{2}+E_{Q}
$$

where $[1,2]$

$$
a=26.98153863(12)
$$

is the relative atomic mass of the neutral aluminum atom,

$$
m_{u} c^{2}=931.494061(21) \mathrm{MeV}
$$

is the mass energy equivalent of the atomic mass constant, and

$$
m_{e} c^{2}=0.510998928(11) \mathrm{MeV}
$$

is the electron mass energy equivalent. The binding energy $E_{Q}$ is the energy required to remove $Q$ electrons from the neutral atom. As shown in 
Section 18 this amounts to $0.322 \mathrm{KeV}$ for $Q=5$ and $6.592 \mathrm{KeV}$ for the fully stripped ion $(Q=13)$.

Thus the mass energy equivalents for the Al5+ and Al13+ ions are

$$
m c^{2}(\mathrm{Al} 5+)=25.1305883178 \mathrm{GeV}
$$

and

$$
m c^{2}(\mathrm{Al} 13+)=25.1265065964 \mathrm{GeV} \text {. }
$$

\section{Kinetic Parameters}

In a circular accelerator the ion moves along an orbit of circumference $C$ with revolution frequency $f$. The radius of the orbit is defined to be $R=C /(2 \pi)$. The velocity of the ion is then

$$
v=2 \pi R f
$$

This gives momentum, energy, and kinetic energy

$$
p=m c \beta \gamma, \quad E=m c^{2} \gamma, \quad W=m c^{2}(\gamma-1)
$$

where

$$
\beta=v / c, \quad \gamma=1 / \sqrt{1-\beta^{2}} .
$$

The magnetic rigidity of the ion in units of $\mathrm{Tm}$ is

$$
B \rho=k c p / Q
$$

where $k=10^{9} / 299792458$ and $c p$ is given in units of $\mathrm{GeV}$. The angular frequency is

$$
\omega=2 \pi f .
$$

We also define the phase-slip factor

$$
\eta=\frac{1}{\gamma_{t}^{2}}-\frac{1}{\gamma^{2}}
$$

where $\gamma_{t}$ is the transition gamma. Note that as defined here, $\eta$ is negative below transition and positive above transition. 


\section{RF Parameters}

1. The stationary bucket area is

$$
A_{S}=8 \frac{R_{s}}{h c}\left\{\frac{2 e Q V_{g} E_{s}}{\pi h\left|\eta_{s}\right|}\right\}^{1 / 2}
$$

where $h$ is the RF harmonic number, $V_{g}$ is the total RF gap voltage per turn, and the subscript " $s$ " denotes parameter values for the synchronous particle.

2. The half-height of a bucket is

$$
\Delta E=\left(\frac{h \omega_{s}}{8 \sqrt{2}}\right) A_{S}\left|\left(\pi-2 \phi_{s}\right) \sin \phi_{s}-2 \cos \phi_{s}\right|^{1 / 2}
$$

where $\phi_{s}$ is the synchronous phase.

3. The synchronous phase is given by

$$
V_{g} \sin \phi_{s}=2 \pi R_{s} \rho_{s} \dot{B} / c
$$

where $\rho_{s}$ is the radius of curvature, $B$ is the magnetic field and $\dot{B}=d B / d t$. Employing Gaussian units $\left(R_{s}\right.$ and $\rho_{s}$ in $\mathrm{cm}$, $c=2.99792458 \times 10^{10} \mathrm{~cm} / \mathrm{s}$, and $\dot{B}$ in $\left.\mathrm{G} / \mathrm{s}\right)$ gives $V_{g} \sin \phi_{s}$ in Statvolts. Multiplying by 299.792458 then gives $V_{g} \sin \phi_{s}$ in Volts.

4. The width of a bucket is

$$
\Delta t=\frac{\left|\pi-\phi_{s}-\phi_{e}\right|}{h \omega_{s}}
$$

where the phase $\phi_{e}$ satisfies

$$
\cos \left(\pi-\phi_{s}\right)-\cos \phi_{e}=-\left(\pi-\phi_{s}-\phi_{e}\right) \sin \phi_{s} .
$$

5. The area of a bucket is

$$
A_{\mathrm{bk}}=\alpha\left(\phi_{s}\right) A_{S}
$$

where

$$
\alpha\left(\phi_{s}\right)=\frac{\sqrt{2}}{8} \int_{\phi_{L}}^{\phi_{R}}\left|\left(\pi-\phi_{s}-\phi\right) \sin \phi_{s}-\cos \phi_{s}-\cos \phi\right|^{1 / 2} d \phi .
$$


Below transition we have $\phi_{e}<\pi-\phi_{s}$ and the limits of integration are $\phi_{L}=\phi_{e}$ and $\phi_{R}=\pi-\phi_{s}$. Above transition we have $\pi-\phi_{s}<\phi_{e}$ and the limits of integration are $\phi_{L}=\pi-\phi_{s}$ and $\phi_{R}=\phi_{e}$. The integral $\alpha\left(\phi_{s}\right)$ must be evaluated numerically. An approximate expression is [3]

$$
\alpha\left(\phi_{s}\right) \approx \frac{1-\sin \phi_{s}}{1+\sin \phi_{s}}
$$

6. The synchrotron frequency for small-amplitude oscillations about $\phi_{s}$ is

$$
F_{s}=\frac{c}{2 \pi R_{s}}\left\{\frac{-h \eta_{s} e Q V_{g} \cos \phi_{s}}{2 \pi E_{s}}\right\}^{1 / 2}
$$

and the corresponding synchrotron tune is $Q_{s}=2 \pi F_{s} / \omega_{s}$. Note that measurement of $F_{s}$ gives a value for $V_{g} \cos \phi_{s}$, while measurement of $d B / d t$ gives a value for $V_{g} \sin \phi_{s}$. These two can be used to obtain $V_{g}$ and $\phi_{s}$.

7. Let $\phi_{l}$ and $\phi_{r}$ be the phases at the left and right boundries of a bunch matched to a bucket. We have

$$
\phi_{l}<\phi_{s}<\phi_{r}
$$

and the width of the bunch is

$$
\Delta t=\frac{\Delta \phi}{h \omega_{s}}, \quad \Delta \phi=\phi_{r}-\phi_{l}
$$

In terms of $\Delta \phi$ and $\phi_{s}$ we have

$$
\phi_{r}=\frac{\Delta \phi}{2}+\arcsin \left\{\frac{\Delta \phi \sin \phi_{s}}{2 \sin (\Delta \phi / 2)}\right\}
$$

and

$$
\phi_{l}=-\frac{\Delta \phi}{2}+\arcsin \left\{\frac{\Delta \phi \sin \phi_{s}}{2 \sin (\Delta \phi / 2)}\right\} .
$$

If $\Delta \phi$ is small we have

$$
\sin (\Delta \phi / 2) \approx \frac{\Delta \phi}{2}, \quad \frac{\Delta \phi \sin \phi_{s}}{2 \sin (\Delta \phi / 2)} \approx \sin \phi_{s}
$$

and

$$
\phi_{l} \approx \phi_{s}-\frac{\Delta \phi}{2}, \quad \phi_{r} \approx \phi_{s}+\frac{\Delta \phi}{2} .
$$


8. The half-height of a bunch matched to a bucket is

$$
\Delta E=\left(\frac{h \omega_{s}}{8 \sqrt{2}}\right) A_{S}\left|\cos \phi_{r}-\cos \phi_{s}+\left(\phi_{r}-\phi_{s}\right) \sin \phi_{s}\right|^{1 / 2}
$$

9. The area of a bunch matched to a bucket is

$$
A_{\mathrm{b}}=F\left(\phi_{s}, \Delta \phi\right) A_{S}
$$

where

$$
F\left(\phi_{s}, \Delta \phi\right)=\frac{\sqrt{2}}{8} \int_{\phi_{l}}^{\phi_{r}}\left|\cos \phi_{l}-\cos \phi+\left(\phi_{l}-\phi\right) \sin \phi_{s}\right|^{1 / 2} d \phi .
$$

The integral $F\left(\phi_{s}, \Delta \phi\right)$ must be evaluated numerically. If $\Delta \phi$ is small we have

$$
F\left(\phi_{s}, \Delta \phi\right) \approx \frac{\pi}{64}(\Delta \phi)^{2}\left|\cos \phi_{s}\right|^{1 / 2} .
$$

\section{Ring Parameters}

\begin{tabular}{|c|c|c|c|c|}
\hline Parameter & Booster & AGS & RHIC & Unit \\
\hline$C_{I}$ & $C_{b}$ & $C_{a}$ & $C_{r}+\delta C$ & $\mathrm{~m}$ \\
\hline$C_{E}$ & $C_{a} / 4$ & $4\left(C_{r}+\delta C\right) / 19$ & $C_{r}+\delta C$ & $\mathrm{~m}$ \\
\hline$\rho$ & 13.8656 & 85.378351 & 242.7806 & $\mathrm{~m}$ \\
\hline$\gamma_{\mathrm{tr}}$ & 4.832 & 8.5 & 22.89 & \\
\hline
\end{tabular}

Here $C_{I}$ and $C_{E}$ are the circumferences of the closed orbits in the machines at injection and extraction (or store) respectively. $C_{b}, C_{a}$, and $C_{r}$ are the circumferences of the "design" orbits in Booster, AGS, and RHIC respectively. These are

$$
C_{b}=201.780, \quad C_{a}=2 \pi(128.4526), \quad C_{r}=3833.845181
$$

meters. $\delta C$ is the shift (if any) of the RHIC orbit circumference from the design value $C_{r}$. Note that $4\left(C_{r} / 19\right)=2 \pi(128.4580) \mathrm{m}$ which gives an AGS radius at extraction approximately $5 \mathrm{~mm}$ larger than the "design" AGS radius (128.4526 m) reported by Bleser [4, 5]. The radius of curvature $\rho$ in the Booster and AGS main dipoles is given in Refs. $[4,5,6]$. The RHIC ring parameters are taken from Ref. [7] and from MAD runs by Steve Tepikian. 


\section{$5 \quad$ Initial Conditions and Assumptions}

1. The revolution frequency of the Al5 + ion (from EBIS) at Booster injection is $96.640 \mathrm{kHz}$. The radius is taken to be the nominal radius $C_{b} /(2 \pi)$.

2. The revolution frequency of the Al5+ ion at Booster extraction is $f=654.157 \mathrm{KHz}$. The radius is taken to be one fourth the nominal AGS radius $C_{a} /(2 \pi)$. The corresponding magnetic rigidity is $B \rho=8.22102484908 \mathrm{Tm}$. The rigidity that can be extracted from Booster into the BTA line is limited by the F3 extraction kicker. The advertised limit is $B \rho=9.5 \mathrm{Tm}[8]$.

3. The set revolution frequency of the Al13+ ion at AGS injection is $f=163.125 \mathrm{KHz}$. This gives an energy loss of $0.63 \mathrm{MeV}$ per nucleon in the BTA stripper. (This number was obtained by Peter Thieberger using the SRIM code.)

4. The magnetic rigidity of the Al13+ ion at RHIC injection is taken to be $B \rho=81.11378003 \mathrm{Tm}$.

5. The circumference shift in RHIC yellow ring at Al13+ injection is $\delta C=-1.9045 \mathrm{~mm}[9]$.

6. The circumference shift in RHIC yellow ring at PP injection is $\delta C=-5.1767 \mathrm{~mm}[9]$.

7. The circumference shift in RHIC yellow ring at Store is $\delta C=-5.120372 \mathrm{~mm}[9]$.

8. The magnetic rigidity of the Al13+ ion in RHIC at PP injection is $B \rho=162.954345233 \mathrm{Tm}[9]$.

9. The magnetic rigidity of the Al13+ ion at RHIC store is $B \rho=683.161277225 \mathrm{Tm}[9]$.

The parameter values given in the following sections are calculated with these initial conditions and assumptions. For many of the parameters more digits are given than would be warranted by the precision with which the parameter could be measured; this is done for computational convenience. 


\section{Longitudinal Emittance of Unbunched Beam in Booster at Injection}

The longitudinal emittance per nucleon of unbunched beam in Booster at injection is

$$
\mathcal{E}=\frac{2}{A} \Delta E \Delta T
$$

where $\Delta E$ is the energy half-width of the beam,

$$
\Delta T=\frac{1}{f}=\frac{2 \pi R}{c \beta}
$$

is the revolution period, and $A$ is the number of nucleons. Using the differential relation

$$
\Delta E=\beta^{2} \frac{\Delta p}{p} m c^{2} \gamma
$$

we have

$$
\mathcal{E}=\frac{2 \beta^{2} \gamma}{f} \frac{m c^{2}}{A} \frac{\Delta p}{p}
$$

where $\Delta p$ is the momentum half-width of the unbunched beam. Taking

$$
f=96.640 \mathrm{kHz}
$$

gives

$$
\begin{gathered}
\Delta T=10.3476821192 \mu \mathrm{s} \\
\beta=0.0650450626079, \quad \gamma=1.00212216641
\end{gathered}
$$

and

$$
\frac{2 \beta^{2} \gamma}{f}=87.7450074295 \mathrm{~ns}
$$

For Al5+ ions we have

$$
\frac{m c^{2}}{A}=0.930762530291 \mathrm{GeV}
$$

which gives

$$
\frac{2 \beta^{2} \gamma}{f} \frac{m c^{2}}{A}=81.6697651354 \mathrm{eV} \mathrm{s}
$$

Taking the fractional momentum half-width to be at most

$$
\frac{\Delta p}{p}=0.001
$$


then gives longitudinal emittance (per nucleon)

$$
\mathcal{E}=0.0816697651354 \mathrm{eV} \mathrm{s} .
$$

The bunch merging schemes used in Booster and AGS put 4 Booster loads into each AGS bunch. The minimum longitudinal emittance of that bunch would then be

$$
4 \mathcal{E}=0.32668 \mathrm{eV} \mathrm{s}
$$

\section{Minimum RF Voltage Required to Capture the Unbunched Beam}

In order to capture the unbunched beam into $h$ buckets we must have RF voltage $V_{g}$ (i.e. total gap voltage per turn) such that

$$
\mathcal{E} \leq \frac{h A_{S}}{A}
$$

where $A_{S}$ is given by (14). Thus we must have

$$
\frac{2 \beta^{2} \gamma}{f} \frac{m c^{2}}{A} \frac{\Delta p}{p} \leq \frac{8 R}{c A}\left\{\frac{2 e Q V_{g} E}{\pi h|\eta|}\right\}^{1 / 2}
$$

which gives

$$
\begin{gathered}
2 \beta^{2} \gamma\left(\frac{2 \pi R}{c \beta}\right) \frac{m c^{2}}{A} \frac{\Delta p}{p} \leq \frac{8 R}{c}\left(\frac{2 \gamma}{\pi h|\eta|}\right)^{1 / 2} \frac{m c^{2}}{A}\left(\frac{e Q V_{g}}{m c^{2}}\right)^{1 / 2} \\
\beta^{2} \gamma\left(\frac{\pi}{\beta}\right) \frac{\Delta p}{p} \leq 2\left(\frac{2 \gamma}{\pi h|\eta|}\right)^{1 / 2}\left(\frac{e Q V_{g}}{m c^{2}}\right)^{1 / 2} \\
\beta^{2} \gamma^{2} \pi^{2}\left(\frac{\Delta p}{p}\right)^{2} \leq\left(\frac{8 \gamma}{\pi h|\eta|}\right)\left(\frac{Q}{m c^{2}}\right) e V_{g}
\end{gathered}
$$

and

$$
\frac{1}{8} h \pi^{3} \beta^{2} \gamma|\eta|\left(\frac{m c^{2}}{Q}\right)\left(\frac{\Delta p}{p}\right)^{2} \leq e V_{g}
$$

Here

$$
h=4
$$


and taking revolution frequency

$$
f=96.640 \mathrm{kHz}
$$

we have

$$
\eta=-0.952939329734
$$

and

$$
\frac{1}{8} h \pi^{3} \beta^{2} \gamma|\eta|=0.0626374709945
$$

For the $\mathrm{Al} 5+$ ion we have mass energy equivalent per unit charge

$$
\frac{m c^{2}}{Q}=5.02611766357 \mathrm{GeV}
$$

Taking fractional momentum half-width

$$
\frac{\Delta p}{p}=0.001
$$

then gives

$$
314.823299367 \text { volts } \leq V_{g}
$$

\section{Inflector Voltage}

At Booster injection, the voltage $V_{I}$ required for particles with mass $m$, velocity $c \beta$, and charge $e Q$ to follow the nominal trajectory through the inflector is given by

$$
e V_{I}=\frac{G}{R_{I}}\left(\frac{m c^{2}}{Q}\right) \beta^{2} \gamma
$$

Here $G=0.021 \mathrm{~m}$ is the gap between the cathode and septum of the inflector and $R_{I}=8.74123 \mathrm{~m}$ is the radius of curvature along the nominal trajectory. Using the values of $\beta, \gamma$, and $m c^{2} / Q$ given by (40) and (57), we obtain

$$
V_{I}=51.195 \mathrm{kV}
$$

for Al5+ ions from EBIS. Because of an unresolved calibration problem, the actual setpoint for the inflector voltage needs to be

$$
V_{I}(\text { setpoint })=51.968 \mathrm{kV} \text {. }
$$




\section{Booster and AGS Injection Fields}

The nominal magnetic field in the Booster dipoles at injection is

$$
B=(B \rho) / \rho
$$

where $B \rho$ is given by (11) and $\rho$ is the nominal radius of curvature. Writing

$$
B \rho=\frac{10^{9}}{c}\left(\frac{m c^{2}}{Q}\right) \beta \gamma
$$

and using the values of $\beta, \gamma$, and $m c^{2} / Q$ given by (40) and (57), we obtain

$$
B \rho=1.09281576898 \mathrm{Tm} \text {. }
$$

Here we have used the mass energy equivalent $m c^{2}$ in units of $\mathrm{GeV}$ and the velocity of light in units of $\mathrm{m} / \mathrm{s}$. Using

$$
\rho=13.8656 \mathrm{~m}
$$

we then obtain

$$
B=788.148921781 \text { Gauss }
$$

for $\mathrm{Al} 5+$ ions from EBIS.

The magnetic field is measured with a Hall probe and the Booster Gauss Clock. The Hall probe sits in the reference dipole and gives the value of the field at BT0. The Gauss Clock gives the change in field between BT0 and the time of measurement. The measured field is defined to be the field at BT0 plus the field change given by the Gauss Clock.

Similarly, the nominal magnetic field in the AGS dipoles at injection is $B=369.12$ Gauss for the Al13+ ions.

\section{BTA Stripper}

The stripper used to strip aluminum ions in the BTA (Booster-To-AGS) transfer line consists of a $6.45 \mathrm{mg} / \mathrm{cm}^{2}$ aluminum foil followed by a 8.39 $\mathrm{mg} / \mathrm{cm}^{2}$ carbon foil. In Section 17 we use these surface densities to calculate the energy loss of Al13+ ions in the foils. 


\section{AGS Injection Septum Magnet Current}

The field required in the L20 septum magnet is

$$
B=(B \rho) / \rho
$$

where $B \rho$ is the magnetic rigidity of the beam and $\rho=18.625 \mathrm{~m}[11]$ is the radius of curvature of the nominal trajectory through the magnet. The required current is given by

$$
N I=g B / \mu_{0}
$$

where $N=1$ is the number of conductor turns; $g=0.0467 \mathrm{~m}$ [11] is the magnet gap; and $\mu_{0}=4 \pi \times 10^{-7} \mathrm{Tm} / \mathrm{A}$.

For Al13+ ions at injection, the magnetic rigidity is $B \rho=3.15149462610$

Tm. This gives $B=0.169208 \mathrm{~T}$ and $I=6288 \mathrm{~A}$.

For comparison, the magnetic rigidity of polarized protons at AGS

injection is $B \rho=7.205178 \mathrm{Tm}$. This gives $B=0.3869 \mathrm{~T}$ and $I=14380 \mathrm{~A}$.

\section{AGS Injection Kicker Current}

The current required in the A5 kicker is $[10,11]$

$$
I=\frac{B \rho}{K} \sin \phi
$$

where

$$
K=1.8718 \times 10^{-5} \mathrm{Tm} / \mathrm{A}
$$

and

$$
\phi=3.35 \text { milliradians }
$$

is the desired kick angle. Using the calculated values of $B \rho$ at AGS injection we obtain a current of $564.03 \mathrm{~A}$ for Al13+ ions. The maximum available current is $1100 \mathrm{~A}$. 


\section{Aluminum in Booster}

\begin{tabular}{|c|c|c|c|c|}
\hline Parameter & Injection & Merge porch & Extraction & Unit \\
\hline$Q$ & 5 & 5 & 5 & \\
\hline$m c^{2}$ & 25.1305883178 & 25.1305883178 & 25.1305883178 & $\mathrm{GeV}$ \\
\hline$W / A$ & 1.97523297380 & 49.2338483823 & 105.879558420 & $\mathrm{MeV}$ \\
\hline$c p / A$ & 60.6699862083 & 306.714874793 & 456.407638293 & $\mathrm{MeV}$ \\
\hline$E / A$ & 0.932737763264 & 0.979996378673 & 1.03664208871 & $\mathrm{GeV}$ \\
\hline$B \rho$ & 1.09281576898 & 5.52468976347 & 8.22102484908 & $\mathrm{Tm}$ \\
\hline$\beta$ & 0.0650450626079 & 0.312975518550 & 0.440275041178 & \\
\hline$\gamma-1$ & 0.00212216640606 & 0.0528962509555 & 0.113755716387 & \\
\hline$\eta$ & -0.953 & -0.859 & -0.763 & \\
\hline$\epsilon_{H}(95 \%)$ & $12.1 \pi$ & $12.1 \pi$ & $12.1 \pi$ & $\mathrm{mm} \mathrm{mrad}$ \\
\hline$\epsilon_{V}(95 \%)$ & $5.68 \pi$ & $5.68 \pi$ & $5.68 \pi$ & $\mathrm{mm} \mathrm{mrad}$ \\
\hline$h$ & 4 & 1 & 1 & \\
\hline$h f$ & 386.560 & 465.000 & 654.157 & $\mathrm{KHz}$ \\
\hline$R$ & $201.780 /(2 \pi)$ & $201.780 /(2 \pi)$ & $128.4526 / 4$ & $\mathrm{~m}$ \\
\hline
\end{tabular}

Here $\epsilon_{H}$ and $\epsilon_{V}$ are the normalized horizontal and vertical transverse emittances. These follow from the assumption that during injection the horizontal and vertical acceptances in Booster are completely filled. The horizontal and vertical acceptances are $185 \pi$ and $87 \pi \mathrm{mm} \mathrm{mrad}$ (un-normalized) respectively. 


\section{Aluminum in AGS}

\begin{tabular}{|c|c|c|c|c|}
\hline Parameter & Injection & Transition & Extraction & Unit \\
\hline$Q$ & 13 & 13 & 13 & \\
\hline$m c^{2}$ & 25.1265065964 & 25.1265065964 & 25.1265065964 & $\mathrm{GeV}$ \\
\hline$W / A$ & 105.232448673 & 6.97958516567 & 10.8146436224 & $\mathrm{GeV}$ \\
\hline$c p / A$ & 0.454900969049 & 7.85526393621 & 11.7083293855 & $\mathrm{GeV}$ \\
\hline$E / A$ & 1.03584380410 & 7.91019652110 & 11.7452549778 & $\mathrm{GeV}$ \\
\hline$B \rho$ & 3.15149462610 & 54.4202447696 & 81.11378003 & $\mathrm{Tm}$ \\
\hline$\beta$ & 0.439159810823 & 0.993055471537 & 0.996856126802 & \\
\hline$\gamma$ & 1.11307883582 & 8.5000 & 12.6210097366 & \\
\hline$\eta$ & -0.793 & 0.0 & 0.00756 & \\
\hline$\epsilon_{H}(95 \%)$ & $\leq 12 \pi$ & $\leq 12 \pi$ & $\leq 12 \pi$ & $\mathrm{mm} \mathrm{mrad}$ \\
\hline$\epsilon_{V}(95 \%)$ & $\leq 12 \pi$ & $\leq 12 \pi$ & $\leq 12 \pi$ & $\mathrm{mm} \mathrm{mrad}$ \\
\hline$h$ & 16 & 12 & 12 & \\
\hline$h f$ & 2.610000 & 4.42642071890 & 4.44317772988 & $\mathrm{MHz}$ \\
\hline$R$ & 128.4526 & 128.4526 & 128.457917578 & $\mathrm{~m}$ \\
\hline
\end{tabular}

\section{Aluminum in RHIC}

\begin{tabular}{|c|c|c|c|c|}
\hline Parameter & Injection & PP Injection & Store & Unit \\
\hline$Q$ & 13 & 13 & 13 & \\
\hline$m c^{2}$ & 25.1265065964 & 25.1265065964 & 25.1265065964 & $\mathrm{GeV}$ \\
\hline$W / A$ & 10.8146436224 & 22.6093571067 & 97.6843642139 & $\mathrm{GeV}$ \\
\hline$c p / A$ & 11.7083293855 & 23.5215662255 & 98.6105844676 & $\mathrm{GeV}$ \\
\hline$E / A$ & 11.7452549778 & 23.5399684621 & 98.6149755693 & $\mathrm{GeV}$ \\
\hline$B \rho$ & 81.11378003 & 162.954345233 & 683.161277225 & $\mathrm{Tm}$ \\
\hline$\beta$ & 0.996856126802 & 0.999218255683 & 0.999955472263 & \\
\hline$\gamma$ & 12.6210097366 & 25.2951657262 & 105.967947838 & \\
\hline$\eta$ & -0.00437 & 0.000346 & 0.001820 & \\
\hline$\epsilon_{H}(95 \%)$ & $\leq 10 \pi$ & $\leq 10 \pi$ & $\leq 10 \pi$ & $\mathrm{mm} \mathrm{mrad}$ \\
\hline$\epsilon_{V}(95 \%)$ & $\leq 10 \pi$ & $\leq 10 \pi$ & $\leq 10 \pi$ & $\mathrm{mm} \mathrm{mrad}$ \\
\hline$f$ & 77.9504864891 & 78.1352629775 & 78.1929095058 & $\mathrm{KHz}$ \\
\hline$h$ & 360 & 360 & 360 & \\
\hline$h f$ & 28.0621751361 & 28.1286946719 & 28.1494474221 & $\mathrm{MHz}$ \\
\hline$\delta C$ & -1.9045 & -5.1767 & -5.120372 & $\mathrm{~mm}$ \\
\hline
\end{tabular}




\section{Center-of-Mass Energy for Proton-Ion Collisions in RHIC}

Let $E_{1}$ and $P_{1}$ be the energy and momentum of an ion circulating in RHIC, and let $E_{2}$ and $-P_{2}$ be the energy and momentum of the counter-circulating ion. The counter-circulating ion may be identical to the circulating one or it may be some other kind of ion.

The center-of-mass (CM) mass-energy equivalent, $M c^{2}$, is given by the Lorentz invariant

$$
M^{2} c^{4}=\left(E_{1}+E_{2}\right)^{2}-\left(c P_{1}-c P_{2}\right)^{2}
$$

where

$$
E_{1}=m_{1} c^{2} \gamma_{1}, \quad c P_{1}=m_{1} c^{2} \beta_{1} \gamma_{1}
$$

and

$$
E_{2}=m_{2} c^{2} \gamma_{2}, \quad c P_{2}=m_{2} c^{2} \beta_{2} \gamma_{2}
$$

Thus we have

$$
\begin{gathered}
M^{2}=\left(m_{1} \gamma_{1}+m_{2} \gamma_{2}\right)^{2}-\left(m_{1} \beta_{1} \gamma_{1}-m_{2} \beta_{2} \gamma_{2}\right)^{2} \\
M^{2}=m_{1}^{2}\left(\gamma_{1}^{2}-\beta_{1}^{2} \gamma_{1}^{2}\right)+m_{2}^{2}\left(\gamma_{2}^{2}-\beta_{2}^{2} \gamma_{2}^{2}\right)+2 m_{1} m_{2} \gamma_{1} \gamma_{2}\left(1+\beta_{1} \beta_{2}\right)
\end{gathered}
$$

and

$$
M^{2}=m_{1}^{2}+m_{2}^{2}+2 m_{1} m_{2} \gamma_{1} \gamma_{2}\left(1+\beta_{1} \beta_{2}\right) .
$$

Here we have used the identities

$$
\gamma_{1}^{2}-\beta_{1}^{2} \gamma_{1}^{2}=1, \quad \gamma_{2}^{2}-\beta_{2}^{2} \gamma_{2}^{2}=1
$$

For Proton-Ion collisions we take

$$
\begin{array}{ll}
\beta_{1}=\beta_{p}, & \gamma_{1}=\gamma_{p} \\
\beta_{2}=\beta_{I}, & \gamma_{2}=\gamma_{I}
\end{array}
$$

and

$$
m_{1}=m_{p}, \quad m_{2}=m_{I} / A
$$

where $A$ is the atomic number (number of nucleons) of the ion, and the subscripts $p$ and $I$ refer to the proton and ion respectively. Thus (78) becomes

$$
M^{2}=m_{p}^{2}+\left(\frac{m_{I}}{A}\right)^{2}+2 m_{p}\left(\frac{m_{I}}{A}\right) \gamma_{p} \gamma_{I}\left(1+\beta_{p} \beta_{I}\right)
$$


where

$$
\beta_{p} \gamma_{p}=\left\{\gamma_{p}^{2}-1\right\}^{1 / 2}, \quad \beta_{I} \gamma_{I}=\left\{\gamma_{I}^{2}-1\right\}^{1 / 2}
$$

The mass-energy equivalent of the proton is [2]

$$
m_{p} c^{2}=0.938272046(21) \mathrm{GeV} .
$$

For the Al13+ ion, the atomic number is

$$
A=27
$$

and the mass-energy equivalent is

$$
m_{I} c^{2}=25.1265065964 \mathrm{GeV} .
$$

For polarized protons, the desired values of $\gamma_{p}$ are quantized by the relation

$$
G \gamma_{p}=k+\frac{1}{2}
$$

where $k$ is a non-negative integer and

$$
G=\left(g_{p}-2\right) / 2 .
$$

Here the proton $\mathrm{g}$ factor is [2]

$$
g_{p}=5.585694713(46)
$$

which gives

$$
G=1.79284735650 .
$$

For polarized protons with

$$
k=198
$$

and Al13+ ions with

$$
\gamma_{I}=105.967947838
$$

we then have CM mass-energy equivalent

$$
M c^{2}=202.429782994 \mathrm{GeV} .
$$




\section{Al13 + Energy Loss in the BTA Stripper Foils}

The stripper used to strip aluminum ions consists of a $6.45 \mathrm{mg} / \mathrm{cm}^{2}$ aluminum foil followed by a $8.39 \mathrm{mg} / \mathrm{cm}^{2}$ "glassy" carbon foil $[12,13]$. We can estimate the energy loss in the foils as follows:

The kinetic energy of a proton that has the same velocity as the Al13+ ion just upstream of the aluminum foil is

$$
W_{p}=106.7 \mathrm{MeV} .
$$

The rate of energy loss of a proton passing through the foil with kinetic energy $W_{p}$ is $[14]$

$$
-\frac{d E_{p}}{d x}=5.416 \mathrm{MeV} \mathrm{cm}^{2} / \mathrm{g} .
$$

The rate of energy loss of the Al13+ ion is obtained by scaling the Bethe-Bloch result for protons [15]. Thus

$$
-\frac{d E}{d x}=-Z^{2} \frac{d E_{p}}{d x} \mathrm{~cm}^{2} / \mathrm{g}
$$

where $Z=13$. Multiplying this by the surface density of the aluminum foil $\left(6.45 \mathrm{mg} / \mathrm{cm}^{2}\right)$ gives

$$
\Delta E_{a}=0.2187 \mathrm{MeV} \text { per nucleon. }
$$

This is the energy lost by the Al13+ ion upon passing through the aluminium foil. The kinetic energy of a proton that has the same velocity as the Al13+ ion just downstream of the aluminum foil is then

$$
W_{p}=106.5 \mathrm{MeV} \text {. }
$$

The rate of energy loss of a proton passing through the carbon foil with this kinetic energy is [14]

$$
-\frac{d E_{p}}{d x}=6.193 \mathrm{MeV} \mathrm{cm}^{2} / \mathrm{g} .
$$

Using this result in (97) with $Z=13$, and multiplying by the surface density of the carbon foil $\left(8.39 \mathrm{mg} / \mathrm{cm}^{2}\right)$ gives

$$
\Delta E_{c}=0.3252 \mathrm{MeV} \text { per nucleon. }
$$

The total energy lost upon passing through both foils is then

$$
\Delta E=\Delta E_{a}+\Delta E_{c}=0.5439 \mathrm{MeV} \text { per nucleon. }
$$

This agrees reasonably well with the value $0.63 \mathrm{MeV}$ per nucleon obtained by Peter Thieberger using the SRIM code. 


\section{Atomic Binding Energies}

To obtain the energy required to remove a certain number of electrons from a given atom we follow Brown and Thieberger [16] and use the tables given in Ref. [17]. Here the table numbered $N$ gives the energy required to remove all electrons from atoms consisting of $N$ electrons and $Z$ protons, with $Z$ running from $N$ to 118 . Tables are given for $N=3$ (Lithium-like atoms) through $N=105$ (Dubnium-like atoms).

Let $E_{Q}$ be the energy required to remove the outer $Q$ electrons from a neutral atom containing $Z$ protons, and let $\mathcal{E}_{Z-Q}$ be the energy required to remove the remaining $Z-Q$ electrons. Then we have

$$
E_{Q}=E_{Z}-\mathcal{E}_{Z-Q}
$$

where $E_{Z}$ is the energy required to remove all $Z$ electrons. Here $E_{Z}$ is obtained from the first entry of Table $Z$ and $\mathcal{E}_{Z-Q}$ is obtained from entry $Z$ of Table $Z-Q$.

For the case of the fully stripped aluminum ion we have $Q=Z=13$ and

$$
E_{Z}=6592 \mathrm{eV}
$$

The ion binding energy $E_{Q}$ is qiven in Table $\mathbf{1}$ for various charge states $Q$.

Table 1: Aluminum Ion Binding Energies $E_{Q}$

\begin{tabular}{|c|c|c|c|}
\hline$Q$ & $Z-Q$ & $\mathcal{E}_{Z-Q}(\mathrm{eV})$ & $E_{Q}(\mathrm{eV})$ \\
\hline 4 & 9 & 6423 & 169 \\
5 & 8 & 6270 & 322 \\
6 & 7 & 6082 & 510 \\
10 & 3 & 4831 & 1761 \\
\hline
\end{tabular}




\section{References}

[1] J.S. Coursey, D.J. Schwab, and R.A. Dragoset, "Atomic Weights and Isotopic Compositions", Nuclear Physics Data, Physical Reference Data, www.nist.gov.

[2] P.J. Mohr and B.N. Taylor, "Values of Fundamental Physical Constants", Physical Constants, Physical Reference Data, www.nist.gov.

[3] S.Y. Lee, "Accelerator Physics", World Scientific, 1999, pp. 229-230

[4] E.J. Bleser, "Where are the AGS Magnets", Accelerator Division Technical Note 215, May 20, 1985.

[5] C.J. Gardner, "Notes on Orbit Equations in the AGS", C-A/AP/Note 164, September 2004.

[6] R. Thern, "Booster Dipole Production Measurements", Booster Technical Note 190, March 13, 1991.

[7] W. Fischer and S. Peggs, "RHIC Parameters", Revision of 3/18/97.

[8] W. Zhang, R. Sanders, A. Soukas and J. Tuozzolo, "An Overview of the Fast Injection-Extraction Kicker Systems of the Brookhaven AGS-Booster Complex", PAC99, pp. 1264-1266.

[9] As calculated by Al Marusic. The circumference shifts are due to the unequal rigidities of polarized protons (PP) and Al13+ ions in the DX magnets.

[10] C.J. Gardner, "Determination of the AGS Injection Kicker Strength from Beam Measurements", C-A/AP/Note 91, December 2002.

[11] C.J. Gardner, "AGS Injection with an Additional Kicker in the A10 Straight Section", C-A/AP/Note 217, September 2005.

[12] C.J. Gardner, et al, "Setup and Performance of the RHIC Injector Accelerators for the 2007 Run with Gold Ions", Proceedings of PAC07, pp. 1862-1864.

[13] P. Thieberger, et al, "Improved Gold Ion Stripping at 0.1 and 10 $\mathrm{GeV} /$ nucleon for the Relativistic Heavy Ion Collider", Phys. Rev. ST Accelerators and Beams 11, 011001 (2008). 
[14] M.J. Berger, J.S. Coursey, M.A. Zucker and J. Chang, "Stopping-Power and Range Tables for Electrons, Protons, and Helium Ions", www.nist.gov/physlab/data/star/index.cfm

[15] W.R. Leo, "Techniques for Nuclear and Particle Physics Experiments", Second Revised Edition, Springer-Verlag, 1994, pp. $24-28$.

[16] K.A. Brown, C. Gardner and P. Thieberger, "Rest Mass of Fully Stripped Ions in RHIC: Updated Values", C-A/AP/Note 293, October 2007.

[17] G.C. Rodrigues, P. Indelicato, J.P. Santos, P. Patte, and F. Parente, "Systematic Calculation of Total Atomic Energies of Ground State Configurations", Atomic Data and Nuclear Data Tables 86 (2004) $117-233$. 